\title{
Thermodynamics of Metal Complex Formation in Aqueous Solution. XIII. Enthalpy Measurements on Copper(I) and Silver(I) Halide Systems
}

\author{
STEN AHRLAND, BERNT TAGESSON and DINKO TUHTAR
}

Inorganic Chemistry 1, Chemical Center, University of Lund, P.0.B. 740, S-220 07 Lund, Sweden

In the silver(I) chloride and bromide systems the thermodynamic functions in aqueous solution have been determined for the reactions $\mathrm{Ag}^{+}+j \mathrm{~L}^{-} \rightarrow \mathrm{AgL}_{j}(j-1)-, j=3$ and 4 , and also for the precipitation reactions $\mathrm{Ag}^{+}+\mathrm{L}^{-} \rightarrow$ AgL(s). In the copper(I) halide systems, the corresponding functions have not been possible to determine. Some interesting information about the thermodynamics of these systems has nevertheless been gained, as the functions referring to the reactions $\mathrm{CuCl}_{2}^{-}+$ $\mathrm{Cl}^{-} \rightarrow \mathrm{CuCl}_{3}{ }^{2-}$ and $\mathrm{CuL}_{3}{ }^{\prime}+3 \mathrm{~L}^{\prime \prime} \rightarrow \mathrm{CuL}_{3}{ }^{\prime \prime}+3 \mathrm{~L}^{\prime}, \mathrm{L}=$ $\mathrm{Cl}^{-}, \mathrm{Br}^{-}$and $\mathrm{I}^{-}$, have been determined. These results have been achieved by the investigation of several types of reactions, performed by various calorimetric techniques. The data refer to a perchlorate medium of an ionic strength $I=5 \mathrm{M}$, at $25^{\circ} \mathrm{C}$.

As is expected for these interactions between soft acceptors and soft donors the complexes are all enthalpy stabilized, while the entropy term counteracts their formation. For both silver(I) and copper(I), the formation reactions become more exothermic as the ligand becomes softer, i.e. in the sequence $\mathrm{Cl}^{-}<\mathrm{Br}^{-}<\mathrm{I}^{-}$. This is of course also the order of increasing stability of the complexes. Although increasingly unfavourable, the entropy term evidently cannot neutralize the stabilizing influence exerted by the enthalpy term in the order mentioned.

In order to elucidate the nature of the coordinate bond in metal complexes, it is useful to know all the thermodynamic functions involved in the formation reactions. ${ }^{1-3}$ The free energy changes $\Delta G^{\circ} \beta j$ can be calculated from the stability constants $\beta_{j}$ :

$$
\Delta G^{\circ}{ }_{\beta j}=-R T \ln \beta_{j}
$$

If the enthalpy changes, $\Delta H^{\circ}{ }_{\beta j}$, are measured, the entropy changes $\Delta S^{\circ}{ }_{\beta j}$ can finally be calculated from

$$
\Delta G^{\circ}{ }_{\beta j}=\Delta H^{\circ}{ }_{\beta j}-T \Delta S^{\circ}{ }_{\beta j}
$$

The enthalpy changes can be obtained, either calorimetrically, or from the temperature coefficients of the stability constants. The calorimetric method is generally much to be preferred as it is both more precise and more accurate..$^{4-6}$ With modern calorimeters, measuring precisely even quite small amounts of heat $(\simeq 0.5 \mathrm{~J})$, the calorimetric method also works with small amounts of reactants, i.e. at fairly low concentrations. Nevertheless, the concentration necessary for a calorimetric measurement are still much larger than those demanded by many methods used for the determination of stability constants..$^{6,7}$ This is of course a restriction on the calorimetric determination of $\Delta H^{\circ}{ }_{\beta j}$, which has proved rather serious for the systems in. vestigated here.

The aim of the present investigation is to determine the thermodynamic functions for the formation of the halide complexes of cop. per(I) and silver(I) in aqueous solution, as far as this is experimentally possible. The stability constants for most of the complexes involved have been determined previously. Moreover, for all systems except silver(I) iodide, measurements exist for identical conditions, i.e. for a perchlorate medium of ionic strength $I=5 \mathrm{M}$, at $25{ }^{\circ} \mathrm{C} .{ }^{8}$ The present measurements of $\Delta H^{\circ} \beta j$ therefore refer to these conditions. As before, sodium perchlorate is used as a 
supplementary salt. In all the present measurements, however, a minor part of the medium, viz. $0.1 \mathrm{M}$, is perchloric acid. The com. pelling reason is that such an acidity is necessary for some of the reactions employed. The choice of $0.1 \mathrm{M}$ perchloric acid was natural as the recent determination of the stabilities of the copper(I) chloride and bromide com. plexes refer to that medium.

A medium of high ionic strength is in fact necessary for the calorimetric measurements as sufficiently high solubilities are reached only at fairly high ligand concentrations.

For copper(I), the disproportionation taking place in aqueous solution, in the absence of stabilizing ligands, prevents all measurements involving the use of solutions of the hydrated metal ion. A direct determination of $\Delta H^{\circ}{ }_{\beta j}$ is therefore not feasible and this of course further severely restricts the scope of the investigation as will be demonstrated. As a consequence, the results achieved for the copper(I) systems are not at all as extensive as those achieved for the corresponding silver(I) systems, where this last complication does not exist.

\section{THE REACTIONS INVESTIGATED CALORIMETRICALLY}

Two essentially different techniques have been used for the calorimetric measurements. In the first one, portions of a solution $T$, containing silver(I) or copper(I), is titrated into a solution $S$ in the calorimeter vessel. In the second one, a solid silver(I) or copper(I) compound is dissolved in the solution present in the calorimeter.

In the titration experiments involving silver(I), $T$ has been a pure perchlorate solution while $\mathbf{S}$ contains various concentrations of chloride or bromide. Iodide and thiocyanate have so far been avoided, as the complex forma. tion reactions are rather more complicated in these systems, involving large amourts of polynuclear complexes even at fairly low concentrations. ${ }^{11,12}$ As long as the concentration of chloride, or bromide, is sufficiently high, and that of silver(I) sufficiently low, formation of complexes $\mathrm{AgL}_{j}^{(j-1)}-$ takes place in the solution. As the silver(I) concentration increases, precipitation of $\mathrm{AgL}(\mathrm{s})$ occurs. This happens sooner, the lower the concentration of ligand chosen. From the measurements in homogeneous solution enthalpy changes referring to the reactions

$\mathrm{Ag}^{+}+j \mathrm{~L}^{-} \rightarrow \mathrm{AgL}_{j}^{(j-1)} ; \Delta H^{\circ}{ }^{\circ} ; j=3,4$

can be calculated and from the measurements involving precipitation, the enthalpy change of

$\mathrm{Ag}^{+}+\mathrm{L}^{-} \rightarrow \mathrm{AgL}(\mathrm{s}) ; \Delta H^{\circ}{ }_{\mathrm{pr}}$

The total halide concentration $C_{\mathrm{L}}$ is so high that it is not appreciably changed by the amounts of $\mathrm{AgL}(\mathrm{s})$ actually precipitated. Once the solubility product has been reached, practically all $\mathrm{Ag}^{+}$added therefore reacts according to eqn. (4), and the amount of $\mathrm{AgL}(\mathrm{s})$ formed can easily be calculated. Moreover, reactions according to eqn. (3) cannot take place to any appreciable extent. The whole of the reaction heat measured thus applies to eqn. (4).

In the case of copper(I), on the other hand, $T$ has had a very high concentration of ligand, in order to protect copper(I) from disproportionation, while $S$ has either been the pure perchlorate medium, or a ligand solution of fairly low concentration. During the titration, [L] decreases so that in principle the enthalpy change for

$\mathrm{CuL}_{j-1}{ }^{(j-2)-}+\mathrm{L}^{-} \rightarrow \mathrm{CuL}_{j}{ }^{(j-1)-} ; \Delta H^{\circ}{ }_{j}$

can be determined. In practice, however, it is only possible to investigate the third step of the chloride system. In the bromide and iodide systems, the variation of the ligand number $\bar{n}$ is too slight in the range of ligand concentrations available and for the thiocyanate system, the solubility is too low for reliable calorimetric measurements. 8

The experiments involving the solution of solid silver(I) compounds have been confined to the bromide system, where the conditions seemed especially favourable. The solubility is fairly high and yet polynuclear complexes are formed only in minor amounts. ${ }^{12}$

In the first set of experiments, silver(I) oxide has been dissolved in the pure perchlorate medium

$\mathrm{Ag}_{2} \mathrm{O}(\mathrm{s})+2 \mathrm{H}^{+} \rightarrow 2 \mathrm{Ag}^{+}+\mathrm{H}_{2} \mathrm{O} ; \Delta H^{\circ}{ }_{\mathrm{A}}$

as well as in a strong bromide solution $\left(C_{\mathrm{L}_{\mathrm{L}}}=\right.$ $4.9 \mathrm{M})$, with an acidity $=0.1 \mathrm{M}$

Acta Chem. Scand. A 31 (1977) No. 8 
$\mathrm{Ag}_{2} \mathrm{O}(\mathrm{s})+2 \mathrm{H}^{+}+2 \overline{\mathrm{n}} \mathrm{Br}^{-} \rightarrow 2 \mathrm{AgBr}_{\mathrm{n}}^{-(\mathrm{n}-1)-}+$

$\mathrm{H}_{\mathbf{2}} \mathrm{O} ; \Delta H^{\circ}{ }_{\mathrm{B}}$

Evidently $[(7)-(6)] / 2$ results in

$\mathrm{Ag}^{+}+\bar{n} \mathrm{Br}^{-} \rightarrow \mathrm{AgBr}_{\mathbf{n}}^{-(\bar{n}-1)-} ; \Delta H^{\circ} \mathrm{C}$

The free bromide concentration $\left[\mathrm{L}^{-}\right] \simeq C_{\mathrm{I}_{1}}$ and as the stability. constants are also known, $\bar{n}$ can be calculated.12 The value is in fact just below $4, v i z .3 .87$. The value of $\Delta H^{\circ}$, found in the titration experiments can confidently be used for the small correction needed in order to transform the value of $\Delta H^{\circ} \mathrm{C}$ found into a value of $\Delta H_{\beta_{4}}$, to be compared with that determined as described above.

In the second solution experiment, silver bromide has been dissolved in the $4.9 \mathrm{M}$ bromide solution

$\mathrm{AgBr}(\mathrm{s})+(\bar{n}-1) \mathrm{Br}^{-} \rightarrow \mathrm{AgBr}_{\bar{n}}^{-(\bar{n}-1)-} ; \Delta H^{\circ}{ }_{\mathrm{D}}$

Evidently, $(9)+(4)=(8)$ (if $\mathrm{L}^{-}=\mathrm{Br}^{-}$) and $\Delta H^{\circ}{ }_{\mathrm{C}}=\Delta H^{\circ}{ }_{\mathrm{D}}+\Delta H_{\mathrm{pr}}^{\circ}$. With the same small correction as before, using $\Delta H^{\circ}$, a value of $\Delta H^{\circ}{ }_{\beta_{4}}$ is again obtained, to be compared with the two previous ones.

In the case of copper(I), a reaction corresponding to eqn. (6) is not possible. The counterpart of eqn. (7) has been studied, however

$\mathrm{Cu}_{2} \mathrm{O}(\mathrm{s})+2 \mathrm{H}^{+}+2 \bar{n} \mathrm{~L}^{-} \rightarrow 2 \mathrm{CuL}_{\overline{\mathrm{n}}}^{-(\bar{n}-1)}-+\mathrm{H}_{2} \mathrm{O} ;$

$\Delta H_{\mathrm{E}}^{\circ}$

and moreover for $\mathrm{L}^{-}=\mathrm{Cl}^{-}, \mathrm{Br}^{-}, \mathrm{I}^{-}$. No measurements involving $\mathrm{SCN}^{-}$have been attempted, as this ligand slowly disintegrates in acid solutions, with the formation of $\mathrm{CN}^{-}$, which might cause difficulties, considering the extreme affinity of $\mathrm{CN}^{-}$for copper(I).

For $\mathrm{Cl}^{-},[\mathrm{L}] \simeq C_{\mathrm{I}_{1}}=4.9 \mathrm{M}$ which means ${ }^{8} \bar{n}=$ 2.77. The complex $\mathrm{CuCl}_{3}{ }^{2-}$ thus predominates although $\mathrm{CuCl}_{2}^{-}$is still present in fairly large amounts. As $\Delta \mathrm{H}^{\circ}{ }_{3}$ of the chloride system has been determined by titration calorimetry, however, the change referring to the formation of the third complex can be calculated.

$\mathrm{Cu}_{2} \mathrm{O}(\mathrm{s})+2 \mathrm{H}^{+}+6 \mathrm{~L}^{-} \rightarrow 2 \mathrm{CuL}_{3}{ }^{2-}+\mathrm{H}_{2} \mathrm{O} ;$

$\Delta H^{\circ} \mathbf{B}$

For bromide, $C_{\mathrm{L}_{4}}=4 \mathrm{M}$. The choice between that value and the somewhat higher $4.9 \mathrm{M}$ does not matter, ${ }^{8}$ as in any case $\bar{n} \simeq 3$. Within the limits of error, the value measured thus actually refers to eqn. (11). For iodide, $C_{\mathrm{L}}=$ $4 \mathrm{M}$ corresponds 8 to a value of $\bar{n}=3.27$. In this case, no data are available from titration experiments to bring about that correction to $\bar{n}=3$ which is necessary in order to ensure a direct comparison with the results found for the other halides. Instead, the measurement has been repeated at the lowest iodide con. centration that still produces a sufficiently rapid solution, viz. $C_{\mathrm{M}}=2.5 \mathrm{M}$, corresponding to $\bar{n}=3.18$. From the values of $\Delta H_{E}^{\circ}$ found at the two values of $\bar{n}$, a reasonable value of $\Delta H^{\circ}{ }_{\mathrm{E} s}$ can be estimated.

Once the values of $\Delta H^{\circ}{ }^{3}$ are known for the various systems, the differences

$\left[\Delta H_{\mathrm{E}_{3}}^{\circ}\left(\mathrm{L}^{\prime}\right)-\Delta H_{\mathrm{E}_{3}}^{\circ}\left(\mathrm{L}^{\prime \prime}\right)\right] / 2=\Delta H_{\beta_{3}}^{\circ}\left(\mathrm{L}^{\prime}\right)-$ $\Delta H^{\circ}{ }_{\beta_{3}}\left(\mathrm{~L}^{\prime \prime}\right)$

can be calculated. These differences between the enthalpy changes accompanying the formation of analogous complexes of the three halide ions are highly informative. They refer to the heats evolved in the substitution reactions (charges omitted)

$$
\mathrm{CuL}_{3}{ }^{\prime \prime}+3 \mathrm{~L}^{\prime} \rightarrow \mathrm{CuL}_{3}{ }^{\prime}+3 \mathrm{~L}^{\prime \prime}
$$

It would of course be even more interesting to know the absolute values of $\Delta H^{\circ} \beta_{3}$ but all attempts to arrive at these have so far failed in spite of strenuous efforts.

The first attempt at this was measuring the reaction corresponding to eqn. (6) in a concentrated solution of copper(II), which would ensure the highest possible concentration of $\mathrm{Cu}^{+}$in solution. This process was slow and erratic. The second attempt tried to utilize the oxidation reactions

$\mathrm{Cu}^{+}+1 / 4 \mathrm{O}_{2}+\mathrm{H}^{+} \rightarrow \mathrm{Cu}^{2+}+1 / 2 \mathrm{H}_{2} \mathrm{O} ; \Delta H^{\circ} \mathrm{O}$

and

$\mathrm{CuCl}_{\mathrm{n}}^{-(\bar{n}-1)-}+1 / 4 \mathrm{O}_{2}+\mathrm{H}^{+} \rightarrow \mathrm{Cu}^{2+}+\bar{n} \mathrm{Cl}^{-}+$ $1 / 2 \mathrm{H}_{2} \mathrm{O} ; \Delta H^{\circ}$ OL,

Also the heats measured in these cases, and especially for eqn. (14), were not reproducible enough for the purpose intended.

\section{EXPERIMENTAL}

Chemicals. Red copper (I) oxide was prepared according to Brauer ${ }^{18}$ (p. 755). Electrolytic

Acta Chem. Scand. A 31 (1977) No. 8 
analysis for total copper gave $86.5 \% \mathrm{Cu}(\mathrm{I})+$ $\mathrm{Cu}(\mathrm{II})$. Titrimetric analysis with cerium(IV) gave $86.1 \% \mathrm{Cu}(\mathrm{I})$. Theoretically, the compound should contain $88.8 \% \mathrm{Cu}(\mathrm{I})$. The low copper content is most probably due to moisture which cannot be removed by heating, however, as the oxide will then decompose. The contamination due to $\mathrm{Cu}$ (II) is in any case slight which is the important fact.

Silver oxide was also prepared according to Brauer ${ }^{18}$ (p. 771). Electrolytic analysis gave 93.0\% Ag (theoretically 93.09\%).

Hydrochloric acid and THAM [tris(hydroxy. methyl)aminomethane] used for testing the solution calorimeter were of analytical grade.

Sodium perchlorate (Fluka) was mostly used without further purification. A purified ${ }^{8}$ preparation was necessary only for those solutions that must not contain even a trace of halide, i.e. the pure silver perchlorate solutions.

The copper (I) and the ligand solutions were prepared and analyzed as described, ${ }^{8}$ while silver perchlorate solution was prepared by neutralizing silver oxide with perchloric acid.

Titration calorimetry. The calorimeter is that developed by Grenthe et al. ${ }^{14}$ For shorter periods ( $\lesssim 1 \mathrm{~h}$, sufficient for the measurement of one experimental point), the temperature of the precision thermostat surrounding the calorimeter stayed constant within $\pm 2 \times 10^{-4}$ ${ }^{\circ} \mathrm{C}$. The room temperature had to be kept at $25.0 \pm 0.2^{\circ} \mathrm{C}$.

In the silver measurements the tube deliver. ing the solution $\mathbf{T}$ had its tip above the surface of the solution $\mathrm{S}$, as the tip was clogged if immersed. To make sure that only a tiny droplet could possibly stay out of the solution S, a very thin-walled capillary tip was used.

The copper(I) solutions had to be very carefully protected against air. During the whole titration, nitrogen was therefore bubbled through the solution in the calorimeter vessel. In order to get the right vapour pressure and temperature, the gas was bubbled through $5 \mathrm{M}$ sodium perchlorate and then passed through a heat exchanger, placed in the precision thermostat, before entering the calorimeter solution.
Initially $100 \mathrm{~cm}^{3}$ of solution $\mathrm{S}$ was equilibrated in the calorimeter which takes $\simeq 2 \mathrm{~h}$. The temperature was adjusted so that a steady increase was obtained during the fore-period. Portions of solution T (at most $4 \mathrm{~cm}^{3}$ ) were then added from a piston burette at a rate of $1 \mathrm{~cm}^{3} / \mathrm{min}$. All additions were made at a special "addition temperature" defined as the temperature where mixing of identical solutions causes no change of the temperature. Between each portion added, the calorimeter was cooled, or heated, back to approximately the initial temperature before a new cycle was started.

The titrations were repeated at least once. For heats of the common magnitude of $5 \mathrm{~J}$, the reproducibility was around $1 \%$, for larger heats the relative error was smaller, for smaller heats larger.

The heat equivalent of the calorimetric system, ${ }^{14}$ obtained by electric calibration, was a linear function of the volume added: $\varepsilon_{V}=$ $2.182+2.162 \times 10^{-2}(V-100) \mathrm{J} / \Omega$ for all solutions of $I=5 \mathrm{M}$ (cf. also Ref. 15).

For all calculations referring to titrations performed in homogenous solution, the computer program "Kalori" 16 was used. The errors given in Tables 2 and 3 are three standard deviations, as given by the program.

Heats of dilution. These must be determined in order to obtain the true heats of reaction from the heats actually measured. The determinations are performed by titrations analogous to those just described, but with only one of the reactants present (see, e.g., Ref. 15).

With the range of concentration used, up to $60 \mathrm{mM}$ silver(I) perchlorate, the heat of dilution of $\mathrm{Ag}^{+}$is quite negligible. Likewise, up to the highest concentration of copper(I) used, $65 \mathrm{mM}$, no measureable heat effect is found when a copper(I) chloride solution of $C_{\mathrm{L}}=4.9 \mathrm{M}$ is added to a copper-free chloride solution of the same concentration.

In the present measurements, the concentrations of anions are extraordinarily high, almost 100 times higher than those of silver(I) or copper(I). This applies both to the perchlorate

Table 1. The heats of dilution, in $\mathrm{J}$, of sodium perchlorate and chloride, when a solution $\mathrm{T}$ is added into a solution $\mathrm{S}$. All solutions contain $0.1 \mathrm{M} \mathrm{HClO}_{4}$. Initial volume $100.0 \mathrm{~cm}^{3}$.

Added

volume of

$\mathrm{S}: \quad 1.96 \mathrm{M} \mathrm{NaBr} \quad 4.9 \mathrm{M} \mathrm{NaBr}$

$4.9 \mathrm{M} \mathrm{NaCl}$

$4.9 \mathrm{M} \mathrm{NaClO}_{4}$

$4.9 \mathrm{M} \mathrm{NaClO}_{4}$ $\mathrm{T} / \mathrm{cm}^{\mathbf{8}}$ T: $4.9 \mathrm{M} \mathrm{NaClO}$ 4 $4.9 \mathrm{M} \mathrm{NaClO}_{4} 4.9 \mathrm{M} \mathrm{NaClO}$ 4 $4.9 \mathrm{M} \mathrm{NaCl}$ $3.9 \mathrm{M} \mathrm{NaCl}$

\begin{tabular}{|c|c|c|c|c|c|}
\hline $\begin{array}{r}3 \\
6 \\
9 \\
12 \\
15 \\
18 \\
18 \\
21\end{array}$ & $\begin{array}{l}-0.50 \\
-0.45 \\
-0.41\end{array}$ & $\begin{array}{l}-9.96 \\
-8.96 \\
-8.13 \\
-7.40 \\
-6.74 \\
-6.16 \\
-5.62\end{array}$ & $\begin{array}{l}-5.45 \\
-4.58 \\
-4.06 \\
-3.56 \\
-3.15 \\
-2.76\end{array}$ & $\begin{array}{l}-0.68 \\
-0.94 \\
-1.07 \\
-1.18 \\
-1.28\end{array}$ & $\begin{array}{l}+0.41 \\
+0.23 \\
+0.12 \\
-0.02 \\
-0.10\end{array}$ \\
\hline
\end{tabular}


and the halide ions. In some series, the dilution might therefore be very drastic. Consequently, considerable heat effects might be expected and are in fact also found, Table 1. Very striking is, however, that the heat of dilution found when a concentrated, i.e. $5 \mathrm{M}$, perchlorate solution is added to a chloride solution of about the same concentration is much higher than that of the opposite process, i.e. addition of chloride to perchlorate. The heat evolved at the addition of perchlorate to bromide is even larger than at the addition to chloride, and the difference is considerable; Table 1. Evident. ly, the ion solvation in these concentrated solutions differs very much from that in dilute solutions and depends strongly on the properties of the individual ions.

In extreme cases, the correction due to the heat of dilution is almost half of the value actually measured but generally it is much smaller, often $\simeq 10 \%$.

Reaction solution calorimetry. This calorimeter was also of the isothermal jacket type. Essentially, the design of Sunner and Wadsö ${ }^{17}$ has been followed. As the reactions studied here proceeded too slowly in a calorimeter of the original design, however, some modifications have been introduced in order to achieve a better contact between the reacting phases. It was observed that after the ampoule containing the solid phase had been broken, the heavy solid stayed at the bottom of the vessel where the stirring was not very efficient. This was improved by modifying the construction in such a way that the stirrer could be fixed in a lowered position, close to the bottom of the vessel, once the ampoule had been broken. The stirring was further made more efficient by fixing eight blades on the stirrer, each of an area $\simeq 10 \mathrm{~mm}^{2}$. Four of these blades were in the upper part of the solution acting as a left hand screw forcing the liquid down, four were close to the bottom, acting as a right hand screw forcing the liquid upwards. With this arrangement, the solids were dissolved in 15 to $20 \mathrm{~min}$ which allows a good measurement. A difficulty with this very efficient stirring was, however, that the circulation of glass fragments from the broken ampoule caused an irregular evolution of friction heat that could not be tolerated. This problem was solved by fastening a platinum net $(0.3 \mathrm{~mm}$ wire, $0.7 \times 0.7$ $\mathrm{mm}$ meshes) along the inside of the calorimeter gold-vessel. In this net the fragments were caught. The net should not extend to the bottom of the vessel as this interferes with the stirring of the solid. Instead it is designed like a standing trough with the end $\simeq 7 \mathrm{~mm}$ above the bottom. With this arrangement, the heat produced in connexion with ampoule breaking was no more than $\Delta H_{\mathrm{br}}=-0.06 \pm 0.03 \mathrm{~J}$. This value was found by breaking empty ampoules, as the mean of five experiments.

The ampoules used for introducing the solid were of the standard LKB type. The filling with $\mathrm{Cu}_{2} \mathrm{O}$ was performed in a nitrogen atmosphere. In these experiments, nitrogen was also bubbled through the solution during the reaction. After the ampoule had been broken, the stirrer was moved up and down three times in order to make it easier to catch the glass fragments before it was finally fixed in the lowered position. In other respects, the experiments were conducted as described before.8

The electric calibration gave $\varepsilon=2.035 \mathrm{~J} / \Omega$ for $90 \mathrm{ml}$ of a solution of $I=5 \mathrm{M}$.

Table 2. The silver chloride and bromide systems. Stability constant and thermodynamic functions of the reactions $\mathrm{Ag}^{+}+j \mathrm{~L}^{-} \rightarrow \mathrm{AgL}^{1-i}$ and $\mathrm{AgL}_{3}{ }^{2-}+\mathrm{L}^{-} \rightarrow \mathrm{AgL}_{4}{ }^{3-}$.

\begin{tabular}{|c|c|c|c|}
\hline Ligand $\rightarrow$ & $j$ & $\mathrm{Cl}^{-}$ & $\mathrm{Br}^{-}$ \\
\hline$\beta_{j} / \mathbf{M}^{-i}$ & $\begin{array}{l}3 \\
4\end{array}$ & $\begin{array}{r}(14.0 \pm 1.0) \times 10^{5 a} \\
(2.1 \pm 0.5) \times 10^{5 a}\end{array}$ & $\begin{array}{l}1.2 \times 10^{\circ b} \\
1.6 \times 10^{\circ b}\end{array}$ \\
\hline$-\Delta G^{\circ}{ }_{\beta j} / \mathrm{kJ} \mathrm{mol}^{-1}$ & $\begin{array}{l}3 \\
\mathbf{4}\end{array}$ & $\begin{array}{l}35.1 \pm 0.2 \\
30.6 \pm 0.7\end{array}$ & $\begin{array}{l}51.8 \\
52.6\end{array}$ \\
\hline$-\Delta H^{\circ} \beta j / \mathrm{kJ} \mathrm{mol}^{-1}$ & $\begin{array}{l}3 \\
4\end{array}$ & $\begin{array}{l}39.2 \pm 4.8 \\
62.0 \pm 8.4\end{array}$ & $\begin{array}{l}54.6 \pm 5.2 \\
79.6 \pm 1.1\end{array}$ \\
\hline$-\Delta S^{\circ}{ }_{\beta j} / \mathrm{J} \mathrm{mol}^{-1} \mathrm{~K}^{-1}$ & $\begin{array}{l}3 \\
4\end{array}$ & $\begin{array}{r}14 \pm 16 \\
105 \pm 29\end{array}$ & $\begin{array}{l}9 \pm 18 \\
91 \pm 4\end{array}$ \\
\hline $\begin{array}{l}-\Delta G^{\circ} / \mathrm{kJ} \mathrm{mol}^{-1} \\
-\Delta H^{\circ} / \mathrm{kJ} \mathrm{mol}^{-1} \\
-\Delta S^{\circ}{ }_{4} / \mathrm{J} \mathrm{mol}^{-1} \mathrm{~K}^{-1}\end{array}$ & & $\begin{array}{c}-4.5 \pm 0.7 \\
23 \pm 10 \\
92 \pm 33\end{array}$ & $\begin{array}{l}0.7 \\
25 \pm 6 \\
82 \pm 18\end{array}$ \\
\hline
\end{tabular}

$a$ Ref. 20. $b$ Ref. 12.

Acta Chem. Scand. A 31 (1977) No. 8 
Table 3. The solubility products and the thermodynamic functions for the precipitation of silver halide complexes, at $25^{\circ} \mathrm{C}$.

\begin{tabular}{|c|c|c|c|c|c|}
\hline $\begin{array}{l}\mathrm{L}^{-} \\
I\end{array}$ & $\mathrm{Cl}^{-}$ & $\begin{array}{l}\mathrm{Br}^{-} \\
\mathbf{5}^{\mathrm{a}}\end{array}$ & $\mathrm{Cl}^{-}$ & $\begin{array}{l}\mathrm{Br}^{-} \\
0^{b}\end{array}$ & $\mathrm{~T}^{-}$ \\
\hline$K_{8} \quad M^{2}$ & $(1.14 \pm 0.06) 10^{-10} c$ & $(2.20 \pm 0.010) 10^{-13 d}$ & & & \\
\hline$-\Delta G_{\mathrm{pr}}^{\circ} \mathrm{kJ} \mathrm{\textrm {mol } ^ { - 1 }}$ & $56.76 \pm 0.14$ & $72.26 \pm 0.12$ & 55.65 & $\begin{array}{l}70.21 \\
84.8\end{array}$ & 91.83 \\
\hline$-\Delta S_{\mathrm{pr}}^{\mathrm{p}} \mathrm{JK}^{-1} \mathrm{~mol}^{-1}$ & $20 \pm 3$ & $\begin{array}{c}80.0 \pm 0.0 \\
26 \pm 2\end{array}$ & 33.8 & 49.0 & 64.7 \\
\hline
\end{tabular}

a Present work. ${ }^{b}$ Ref. 21. ${ }^{c}$ From values of $K_{\mathrm{s}}$ determined ${ }^{20}$ at $I=5 \mathrm{M}$ and $I<[\mathrm{Cl}]<2.5 \mathrm{M}$. $d$ From values of $K_{8}$ determined 12 at $I=5 \mathrm{M}$ and $1<\left[\mathrm{Br}^{-}\right]<2.5 \mathrm{M}$.

The accuracy of the calorimeter was tested by dissolving varying amounts $(60-150 \mathrm{mg})$ of THAM in $90 \mathrm{~cm}^{3} 0.100 \mathrm{M}$ hydrochloric acid. A value of $-\Delta H=29.77 \pm 0.11 \mathrm{~kJ} / \mathrm{mol}$ was found, in perfect agreement with the value considered as the "best" one, $10-\Delta H=29.75$ $\mathbf{k J} / \mathbf{m o l}$.

\section{RESULTS}

Silver (I) complexes. The results of the homogeneous titrations are shown in Table 2. For the chloride system, the values of $\Delta H^{\circ}{ }_{\beta j}$ are unfortunately not very precise. This is due to the slight variation of $\bar{n}$ within the range of $C_{\mathrm{L}}$ available and, in the case of $\Delta H^{\circ} \beta_{4}$, also to the poor precision ${ }^{20}$ of $\beta_{4}$. The lowest value of $C_{\mathrm{L}}$ compatible with a sufficiently high solu. bility is $\simeq 1.9 \mathrm{M}$, corresponding to $\bar{n}=3.22$, while even at the highest value of $C_{L_{1}}$ used, 4.6 $\mathrm{M}$, the value of $\bar{n}$ reached is not higher than 3.40. For the bromide systems, where it is possible to use a value of $C_{\mathrm{L}}$ as low as $\simeq 0.5$ $M$, the conditions are more favourable. ${ }^{10,8}$ This lower limit of $C_{\mathrm{I}}$ corresponds to $\bar{n}=3.37$ while at the upper limit of $C_{\mathrm{L}}=4.9 \mathrm{M}, \bar{n}=3.86$. Consequently, the precision of $\Delta H^{\circ} \beta_{4}$ is quite good while that of $\Delta H^{\circ}{ }_{\beta_{3}}$ is still mediocre. The precision of the values of $\Delta S^{\circ} \beta j$ follows, of course, the same pattern, Table 2.

In the range of concentrations investigated here, the values of $\Delta H^{\circ} \beta j$ are independent of $C_{M}$. Consequently, no polynuclear complexes are formed.

The results of the heterogeneous titrations are in Table 3 . In the case of chloride, the value of $\Delta H^{\circ}{ }_{\mathrm{pr}}$ is the mean of seven experiments. In each, $\simeq 30 \mu \mathrm{mol} \mathrm{of} \mathrm{Ag}^{+}$is added to a $2 \mathrm{M}$ chloride solution, already in equilibrium with
$\mathrm{AgCl}(\mathrm{s})$ [cf. eqn. (4)]. In the case of bromide, four experiments were performed by adding $\simeq 90 \mu \mathrm{mol} \mathrm{Ag}+$ to a $2 \mathrm{M}$ bromide solution, and four by adding $\simeq 30 \mu \mathrm{mol} \mathrm{Ag}^{+}$to a $1 \mathrm{M}$ bromide solution. The value of $\Delta H^{\circ}{ }_{p r}$ is the mean of all eight experiments. As the precipitation reactions are simple and complete and the values of $K_{\mathrm{s}}$ well-known, all the thermodynamic functions can be calculated with a rather high degree of precision.

The thermodynamics of the precipitation of the silver halides has been frequently studied in the past. Recently, a very careful determination of $\Delta H^{\circ}$ pr for $\mathrm{AgCl}, \mathrm{AgBr}$ and $\mathrm{AgI}$ has been performed by Wagman and Kilday." With a reaction solution calorimeter, they measured the heats of transforming $\mathrm{AgNO}_{3}(\mathrm{~s})$ into $\mathrm{AgL}(\mathrm{s})$ by means of potassium halide solutions and also the heats of the reciprocal reactions, transforming $\mathrm{KL}(\mathrm{s})$ into $\mathrm{AgL}(\mathrm{s})$ by means of a silver nitrate solution. Combined with the heats of solution of $\mathrm{AgNO}_{3}(\mathrm{~s})$ and $\mathrm{KL}(\mathrm{s})$, respectively, which were also measured, these heats of transformation yield $\Delta H^{\circ}{ }_{\mathrm{pr}}$. Very nearly the same values are obtained by the two approaches. The values are corrected to $I=0$. Values of $\Delta G_{\mathrm{pr}}^{\circ}$ at $I=0$ are selected and corresponding values of $\Delta S_{\mathrm{pr}}^{\circ}$ calculated. The functions thus found are listed in Table 3. The differences between the values of $\Delta H_{\mathrm{pr}}^{\circ}$ determined at $I=$ 0 and $5 \mathrm{M}$ are modest, $\simeq 4 \mathrm{~kJ} \mathrm{~mol}{ }^{-1}$ for both the chloride and the bromide system.

Wagman and Kilday also give an extensive survey of previous investigations of the thermodynamics of silver halide precipitation. These include the classical calorimetric measurements of $\Delta H_{\mathrm{pr}}^{\circ}$ by Berthelot ${ }^{22,23}$ and by Thomsen, and also the very early potentiometric deter- 
Table 4. Silver bromide system. Determination of $\Delta H^{\circ} \beta_{s}$, by reaction solution calorimetry. Values of $\Delta H^{\circ}$ in $\mathrm{kJ} \mathrm{mol}^{-1}$.

\begin{tabular}{|c|c|c|c|c|}
\hline Solid & $-\Delta H_{\mathrm{A}}^{\circ}$ & $-\Delta H_{\mathbf{B}}^{\circ}$ & $-H^{\circ} \mathrm{C}$ & $-H^{\circ} \beta_{4}$ \\
\hline $\begin{array}{l}\mathrm{Ag}_{2} \mathrm{O} \\
\mathrm{AgBr}\end{array}$ & $54.7 \pm 1.3$ & $\begin{array}{r}213.8 \pm 1.5 \\
\Delta H^{\circ}{ }_{D}=3.9 \pm 0.5\end{array}$ & $\begin{array}{l}79.5 \pm 1.4 \\
76.1 \pm 1.0\end{array}$ & $\begin{array}{l}82.8 \pm 2.2 \\
79.3 \pm 1.8\end{array}$ \\
\hline
\end{tabular}

minations of $\Delta G^{\circ}{ }_{\mathrm{pr}}$ by Goodwin, ${ }^{25}$ all resulting in values close to those accepted today.

In Table 4, the results of the solution of $\mathrm{Ag}_{2} \mathrm{O}(\mathrm{s})$ and $\mathrm{AgBr}(\mathrm{s})$ are presented. In the case of $\mathrm{Ag}_{2} \mathrm{O}$, both $\Delta H_{\mathrm{A}}^{\circ}$ and $\Delta H_{\mathrm{B}}^{\circ}$ are the mean values of six experiments where 65 to $200 \mu \mathrm{mol}$ have been dissolved in $0.1 \mathrm{M}$ acid, or in $4.9 \mathrm{M}$ bromide solution of an acidity $=0.1 \mathrm{M}$, respectively. In the case of $\mathrm{AgBr}, \Delta H^{\circ}$ is the mean of five experiments where 130 to $370 \mu \mathrm{mol}$ have been dissolved in the bromide solution. As the value of $\Delta H_{\mathrm{pr}}^{\circ}$ refers to the formation of wet $\operatorname{AgBr}(\mathrm{s})$, the values actually measured at the solution of dry $\mathrm{AgBr}(\mathrm{s})$ have been corrected for the enthalpy of wetting. The correction is quite small, however, viz. $-0.45 \pm$ $0.06 \mathrm{~kJ} \mathrm{~mol}^{-1}$. Moreover, as $\bar{n}=3.87$ is so close to 4 , the correction from $\Delta H^{\circ}{ }_{\mathrm{C}}$ to $\Delta H^{\circ}{ }_{\beta}$, does not introduce any large error, in spite of the rather large error of $\Delta \mathrm{H}^{\circ}$, Table 2.

For the bromide system, the value of $\Delta H^{\circ} \beta_{4}$ has thus been determined by three different calorimetric methods. The results agree in a very satisfactory manner, Table 5. A somewhat higher precision is achieved by titration than by solution calorimetry, but the difference is by no means large. The modified reaction solution calorimeter works well and is evidently able to handle also reactions which are too slow for the old design.

As pointed out earlier, the slightly soluble silver halide systems are not especially well suited to calorimetric work. Indeed they exemplify systems where determination of the thermodynamic functions by means of the temperature coefficient method might be competitive. Such an investigation of the silver bromide system has in fact recently been performed. ${ }^{26}$ The results, referring to a $4.6 \mathrm{M}$ sodium nitrate medium, are also listed in Table 5. As might be expected the drastic qualitative change of the medium markedly influences the stability constants. Much the same value of $\Delta H^{\circ} \beta_{3}$ is found as in the present

Table 5. The silver(I) bromide system. Comparison of the thermodynamic functions found by different methods, at $25^{\circ} \mathrm{C}$.

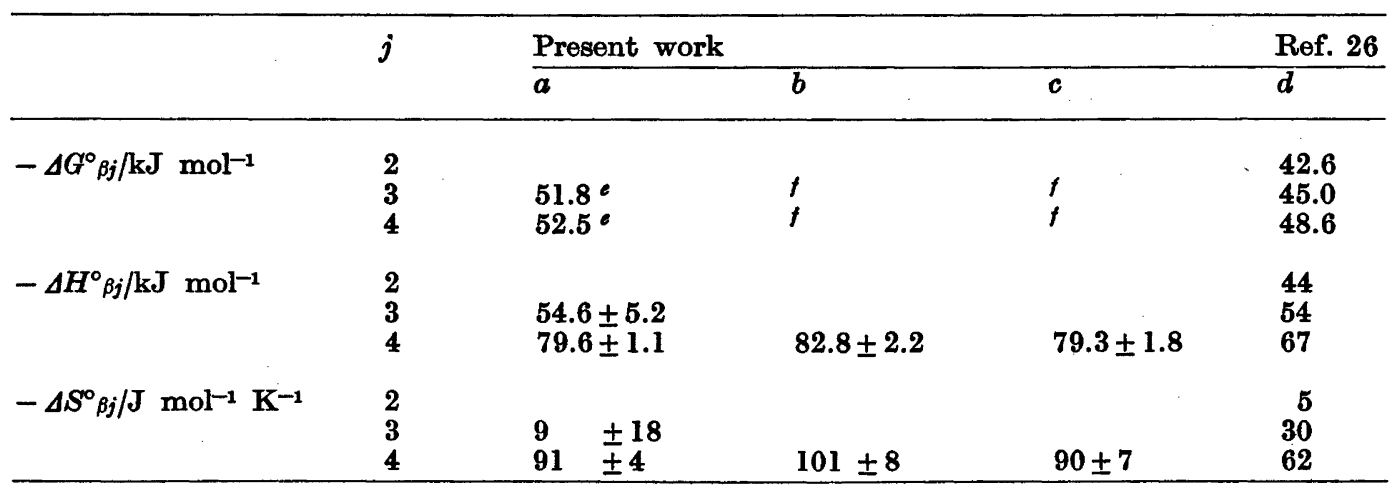

a Titration calorimetry. ${ }^{b}$ Solution of $\mathrm{Ag}_{2} \mathrm{O}(\mathrm{s})$. ${ }^{c}$ Solution of $\mathrm{AgBr}(\mathrm{s})$ combined with precipitation of $\mathrm{AgBr}(\mathrm{s})$. $d$ In 4.6 M NaNO${ }_{3}$; from the temperature coefficient of $\beta_{j}$. ${ }^{e}$ Ref. $12 .{ }^{t}$ The same values of $\Delta G_{\beta j}$ (see note $e$ ) have been used for all calculations referring to the present work.

Acta Chem. Scand. A 31 (1977) No. 8 
Table 6. Copper(I) chloride system. The thermodynamic functions for the reaction $\mathrm{CuCl}_{2}-+$ $\mathrm{Cl}^{-} \rightarrow \mathrm{CuCl}_{3}^{2-}$, at $25^{\circ} \mathrm{C}$.

\begin{tabular}{cccc}
\hline$I \rightarrow$ & $5^{a}$ & $5^{b}$ & $2^{b}$ \\
\hline$K_{3} \mathrm{M}^{-1}$ & $0.76 \pm 0.10$ & 1.3 & 2.6 \\
$-\Delta G^{\circ} \mathrm{kJ} \mathrm{mol}^{-1}$ & $-0.7 \pm 0.2$ & 0.7 & 2.4 \\
$-\Delta H^{\circ} \mathrm{kJ} \mathrm{mol}^{-1}$ & $19.9 \pm 0.7$ & 7.7 & 11.2 \\
$-\Delta S^{\circ}{ }_{3} \mathrm{~J} \mathrm{~K}^{-1} \mathrm{~mol}^{-1}$ & $69 \pm 3$ & 23 & 30 \\
\hline
\end{tabular}

a Present work, sodium chloride-perchlorate medium, acidity $=0.1 \mathrm{M}^{b}$ Ref. 27, hydrochloric-perchloric acid medium.

investigation while the value of $\Delta H^{\circ}{ }_{\beta_{4}}$ differs considerably.

Copper(I) complexes. Titration calorimetry has been possible only in the case of the chloride system, cf. eqn. (5). The value of $C_{\mathrm{L}}$ has been varied between 0.49 and $4.87 \mathrm{M}$, corresponding to a variation of $\bar{n}$ between 2.23 and 2.77. With the seemingly reliable value of $K_{8}$ available, ${ }^{8}$ the data allow the calculation of a good value of $\Delta H^{\circ}{ }_{3}$, listed in Table 6 , together with the corresponding values of $\Delta G^{\circ}{ }_{3}$ and $\Delta S^{\circ}{ }_{3}$.

Within the range of $C_{\mathrm{M}}$ used $\Delta H^{\circ}{ }_{8}$ is independent of $C_{M}$ which implies that the formation of polynuclear complexes is insignificant at these concentrations of copper(I). This is in accordance with the results of the stability measurements. ${ }^{\circ}$

Recently, ${ }^{27}$ values of $\Delta H^{\circ}$ for the copper(I) chloride system have also been determined by dissolving $\mathrm{CuCl}(\mathrm{s})$ in hydrochloric acid of varying concentration, at 15,25 and $35{ }^{\circ} \mathrm{C}$. Media of $I=2,3,4$ and 5 are brought about by means of perchloric acid. Values of $\Delta G^{\circ}$ s are selected for the various media and temperatures used, and corresponding values of $\Delta S^{\circ}{ }_{3}$ calculated. In Table 6, the values referring to $I=2 \mathrm{M}$ and $5 \mathrm{M}$, at $25^{\circ} \mathrm{C}$, have been listed. They differ considerably from our results. Partly, this is certainly due to the large qualitative difference of medium. It might also be, however, that the values of $K_{8}$ selected are not the most probable ones. If so, the values of $\Delta H^{\circ}{ }_{3}$ might easily get rather far off the mark, as they are quite sensitive to changes in the values of $K_{3}$.

The results of the solution of $\mathrm{Cu}_{2} \mathrm{O}(\mathrm{s})$ are presented in Table 7. Each value of $\Delta H^{\circ}$ is the mean of four or five determinations. In the chloride and bromide solutions, 70 to $280 \mu \mathrm{mol}$ have been dissolved in each experiment, in the iodide solutions only 30 to $60 \mu \mathrm{mol}$. Less oxide was used in the latter case in order to avoid the formation of polynuclear complexes which are formed more readily in the iodide system. ${ }^{8}$ In the present solutions, their concentrations should be $<2 \%$. That only insignificant amounts are formed is also proved by the fact that the values of $\Delta H^{\circ}{ }_{\mathrm{E}}$ found are independent of $C_{\mathrm{M}}$.

For the chloride system, the precise value of $\Delta H^{\circ}{ }_{8}$ obtained by titration calorimetry permits the calculation of a good value of $\Delta H^{\circ}{ }_{\mathrm{E} 3}$. As to the bromide system, $\bar{n} \simeq 3$ and consequently $\Delta H_{\mathrm{E}}^{\circ} \simeq \Delta H_{\mathrm{E}}{ }^{\circ}$. Also this quantity has a small random error. For the iodide system, finally, the random errors are so large that no significant difference was found between the values of $\Delta H_{E}^{\circ}$ measured at $\bar{n}=3.18$ and 3.27. This shows, on the other hand, that $\Delta H^{\circ}{ }^{\circ}$ must have much the same value, admittedly only within the fairly wide limits of error.

From the values of $\Delta H^{\circ}{ }_{E s}$ thus found, the differences $\Delta H^{\circ} \beta_{3}\left(\mathrm{~L}^{\prime}\right)-\Delta H^{\circ} \beta_{3}\left(\mathrm{~L}^{\prime \prime}\right)$ have been calculated [eqn. (12)]. As the corresponding differences $\Delta G_{\beta_{3}}^{\circ}\left(\mathrm{L}^{\prime}\right)-\Delta G^{\circ} \beta_{3}\left(\mathrm{~L}^{\prime \prime}\right)$ are known

Table 7. Copper(I) halide systems. Dissolution of $\mathrm{Cu}_{2} \mathrm{O}(\mathrm{s})$ in acid halide solutions.

\begin{tabular}{|c|c|c|c|c|}
\hline $\mathbf{L}^{-}$ & $C_{\mathrm{L}} / \mathrm{M}$ & $\bar{n}$ & $\begin{array}{l}-\Delta H^{\circ} \mathrm{F}^{\prime} \\
\mathrm{kJ} \mathrm{mol}^{-1}\end{array}$ & $\begin{array}{l}-\Delta H^{\circ}{ }^{\circ} / \\
\mathrm{kJ} \mathrm{mol}^{-1}\end{array}$ \\
\hline $\begin{array}{l}\mathrm{Cl}^{-} \\
\mathrm{Br}^{-} \\
\mathrm{I}^{-}\end{array}$ & $\begin{array}{l}4.88 \\
3.98 \\
4.00 \\
2.50\end{array}$ & $\begin{array}{l}2.77 \\
2.98 \\
3.27 \\
3.18\end{array}$ & $\begin{array}{r}50.8 \pm 1.2 \\
80.8 \pm 0.5 \\
156.7 \pm 2.5 \\
156.9 \pm 3.5\end{array}$ & $\begin{array}{c}57.7 \pm 1.4 \\
81 \pm 1 \\
156 \pm 5\end{array}$ \\
\hline
\end{tabular}

Acta Chem. Scand. A 31 (1977) No. 8 
Table 8. The copper(I) halide systems. The differences between the thermodynamic functions for the reactions $\mathrm{Cu}^{+}+3 \mathrm{~L}^{-} \rightarrow \mathrm{CuL}_{3}^{2-}, \mathrm{L}=\mathrm{Cl}^{-}, \mathrm{Br}^{-}, \mathrm{I}^{-}$. The values refer to ligand substitution according to $\mathrm{CuL}_{3}^{\prime \prime}+3 \mathrm{~L}^{\prime} \rightarrow \mathrm{CuL}_{3}^{\prime}+3 \mathrm{~L}^{\prime \prime}$.

$$
\begin{array}{ll}
\mathrm{L}^{\prime}=\mathrm{Br}^{-} & \mathrm{L}^{\prime}=\mathrm{I}^{-} \\
\mathrm{L}^{\prime \prime}=\mathrm{Cl}^{-} & \mathrm{L}^{\prime \prime}=\mathrm{Br}^{-}
\end{array}
$$

$\Delta G^{\circ} \beta_{3}\left(\mathrm{~L}^{\prime}\right)-\Delta G^{\circ} \beta_{3}\left(\mathrm{~L}^{\prime \prime}\right) / \mathrm{kJ} \mathrm{mol}^{-1}$

$\Delta H^{\circ} \beta_{3}\left(\mathrm{~L}^{\prime}\right)-\Delta H^{\circ} \beta_{3}\left(\mathrm{~L}^{\prime \prime}\right) / \mathrm{kJ} \mathrm{mol}^{-1}$

$\Delta S^{\circ} \beta_{3}\left(\mathrm{~L}^{\prime}\right)-\Delta S^{\circ} \beta_{3}\left(\mathrm{~L}^{\prime \prime}\right) / \mathrm{J} \mathrm{mol}^{-1} \mathrm{~K}^{-1}$
$-8.61 \pm 0.13$

$-12 \pm 1$

$-11 \pm 3$

$-17.03 \pm 0.12$
$-38 \quad \pm 3$
$-69 \quad \pm 10$

from the stability measurements, $\Delta S^{\circ} \beta_{8}\left(\mathrm{~L}^{\prime}\right)-$ $\Delta S^{\circ}{ }_{\beta_{3}}\left(L^{\prime \prime}\right)$ can also be calculated. The values found have been listed in Table 8.

\section{DISCUSSION AND CONCLUSIONS}

All the reactions studied involving the formation of halide complexes of silver(I) and copper(I) in aqueous solution are without exception exothermic; Tables 2, 5 and 6. Though the data are not complete, it can further be safely assumed that the enthalpy changes become more negative in the order $\mathrm{Cl}^{-}<\mathrm{Br}^{-}<\mathrm{I}^{-}$, cf. Tables 2 and 8 . Also the precipitation of the silver halides follow the same pattern, Table 3 . This behaviour is certainly to be expected for interactions taking place in aqueous solution between soft metal ions, like the present ones, and the heavy halides, the softness of which increases in the order mentioned. The thermodynamics indicates that the bonds are markedly covalent, and the covalent character is strengthened as the ligand becomes softer. ${ }^{20,1,2}$

The entropy changes are also all negative which means that they counteract the complex formation, as well as the precipitation of solid halides, Tables $2,3,5$ and 6 . This is because these monovalent metal ions are so poorly hydrated that the order of the systems is improved by the complex formation, and by the precipitation of crystalline solids. The entropy changes generally become more unfavourable as the ligand increases in size and consequently, becomes less strongly hydrated, ${ }^{28}$ Tables 3 and 8. At the formation of the third and fourth chloride and bromide complexes of silver(I), however, the entropy changes are practically the same for both ligands, Table 2 .

Also when the entropy changes become more negative in the usual sequence this does not at all compensate the simultaneous decrease of the enthalpies. On the balance, a considerable net increase of the stabilities takes place $^{8}$ in the order $\mathrm{Cl}^{-}<\mathrm{Br}^{-}<\mathrm{I}^{-}$, although of course markedly smaller than would follow from the enthalpy changes alone.

Unfortunately, a comparison between the enthalpy changes of analogous reactions of silver(I) and copper(I) is possible only in one case, viz. between the values of $\Delta H^{\circ} \beta_{3}(\mathrm{Br})-$ $\Delta H^{\circ} \beta_{3}(\mathrm{Cl})$, characterizing the reaction of eqn. (13). The values found are for silver(I) $-15 \pm 10$ $\mathrm{kJ}$ and for copper(I) $-12 \pm 1 \mathrm{~kJ}$, Tables 2 and 8. The experimental error of the silver value is large enough to cover a significant difference between the two acceptors but evidently not a very large one. For the divalent $d^{10}$ acceptor mercury(II), with coordinating properties similar to silver(I) and copper(I), this difference is significantly more negative, ${ }^{30}$ viz. $-35 \pm 7 \mathrm{~kJ}$, at $I=3 \mathrm{M}$ and $25^{\circ} \mathrm{C}$. This trend is even more obvious at the formation of the highest complexes $\mathrm{ML}_{4}$ which can be studied in the case of silver(I) and mercury(II). The values of $\Delta H_{\beta_{4}}(\mathrm{Br})-\Delta H_{\beta_{4}}(\mathrm{Cl})$ are $-18 \pm 9 \mathrm{~kJ}$ (Table 2) and $-47 \pm 3 \mathrm{~kJ}$, respectively. ${ }^{30}$

Acknowledgement. We gratefully acknowledge the generous support given to this work by the Swedish Natural Science Research Council.

\section{REFERENCES}

1. Ahrland, S. Struct. Bonding (Berlin) 5 (1968) 118.

2. Ahrland, S. Struct. Bonding (Berlin) 15 (1973) 167.

3. Schwarzenbach, G. Pure Appl. Chem. 24 (1970) 307.

4. McAuley, A. and Nancollas, G. J. Chem. Soc. (1963) 989.

5. Choppin, G. R. and Schneider, J. K. $J$. Inorg. Nucl. Chem. 32 (1970) 3283.

6. de Carvalho, R. G. and Choppin, G. R. $J$. Inorg. Nucl. Chem. 29 (1967) 725, 737.

Acta Chem. Scand. A 31 (1977) No. 8 
7. Ahrland, S. In Lagowski, J. J., Ed., The Chemistry of Non-Aqueous Solvents, Academic, New York 1977, Vol. 5.

8. Ahrland, S. and Tagesson, B. Acta Chem. Scand. A 31 (1977) 615.

9. Ahrland, S. and Rawsthorne, J. Acta Chem. Scand. 24 (1970) 157.

10. Leden, I. Acta Chem. Scand. 10 (1956) 540, 812.

11. Leden, I. and Nilsson, R. Z. Naturforsch. Teil A 10 (1955) 67.

12. Berne, E. and Leden, I. Z. Naturforsch. Teil A 8 (1953) 719.

13. Brauer, G. Handbuch der preparativen anorganischen Chemie, Enke, Stuttgart 1954.

14. Grenthe, I., Ots, H. and Ginstrup, $O$. Acta Chem. Scand. 24 (1970) 1067.

15. Ahrland, S. and Kullberg, L. Acta Chem. Scand. 25 (1971) 3471.

16. Karlsson, R. and Kullberg, L. Chem. Scr. 9 (1976) 54.

17. Sunner, S. and Wadsö, I. Science Tools 13 (1966) 1.

18. Kullberg, L. Acta Chem. Scand. A 28 (1974) 979.

19. Hill, J. O., Öjelund, G. and Wadsö, I. J. Chem. Thermodyn. 1 (1969) 11.

20. Leden, I. Sven. Kem. Tidskr. 64 (1952) 249.

21. Wagman, D. D. and Kilday, M. V. J. Res. Natl. Bur. Stand. Sect. A 77 (1973) 569.

22. Berthelot, M. Ann. Chim. Phys. [5] 4 (1875) 160.

23. Berthelot, M. C. R. Acad. Sci. 93 (1881) 870.

24. Thomsen, J. Thermochemical Investigations, Barth, Leipzig 1883, Vol. 3.

25. Goodwin, H. M. Z. Phys. Chem. 13 (1894) 577.

26. Pouradier, J. and Pailliotet, A. C. R. Acad. Sci. Ser. C 280 (1975) 1049.

27. Vasil'ev, V. P. and Kunin, B. T. Russ. J. Inorg. Chem. 20 (1975) 1050.

28. Morris, D. F. C. Struct. Bonding (Berlin) 4 (1968) 63.

29. Ahrland, S. Helv. Chim. Acta 50 (1966) 306.

30. Arnek, R. Ark. Kemi 26 (1965) 531.

Received May 11, 1977. 